\title{
Blood cell classification using the Hough transform and convolutional neural networks
}

\author{
Miguel A. Molina-Cabello, Ezequiel López-Rubio, Rafael M. Luque-Baena, \\ María Jesús Rodríguez-Espinosa, and Karl Thurnhofer-Hemsi \\ Department of Computer Languages and Computer Science, University of Málaga, \\ Bulevar Louis Pasteur, 35, 29071 Málaga, Spain \\ \{miguelangel, ezeqlr, rmluque, karlkhader\}@lcc.uma.es \\ mjesus.rodriguez.espinosa@hotmail.com
}

\begin{abstract}
The detection of red blood cells in blood samples can be crucial for the disease detection in its early stages. The use of image processing techniques can accelerate and improve the effectiveness and efficiency of this detection. In this work, the use of the Circle Hough transform for cell detection and artificial neural networks for their identification as a red blood cell is proposed. Specifically, the application of neural networks (MLP) as a standard classification technique with (MLP) is compared with new proposals related to deep learning such as convolutional neural networks (CNNs). The different experiments carried out reveal the high classification ratio and show promising results after the application of the CNNs.
\end{abstract}

Keywords: blood cell detection, blood cell classification, circle Hough transform, convolutional neural networks

\section{Introduction}

Digital image processing is of paramount importance in various medicine fields, from images which are obtained in medical tests such as X-ray image, computed tomography, magnetic resonance imaging, ultrasound image and nuclear medicine image, to images which are obtained in laboratory by microscopy. In this way, digital image processing allows to process the image to obtain a better visibility, to emphasize the required parts or to make analysis and predictions. In addition, image segmentation through image processing is essential for pathology detection $[12,4]$.

Nowadays, most of images obtained in laboratory by microscopy are digitalized afterwards and processed by computers to make easier and faster the image analysis process. Among other fields, blood optical microscopy provides image samples whose study can supply very useful information about patient health. The techniques applied in hematology to count blood cells in blood samples, such as centrifugation techniques or hematology cell counters, are imprecise and inexact in many cases. Thus, the result can change significantly according to the measure technique used [3,8]. Moreover, automated cell counters are used 
with less frequency than manual cell counters in laboratories. Thus, laboratory operations might be optimized by automated cell counters, considering manual cell counters for validation purposes [11].

For a long time, many researches have been carried out to develop tools or techniques which reduce the errors in the manual method, the cost and the slowness at blood cells count for pathology detection [9, 14]. In a blood sample, the percentage occupied by red blood cells in relation to the total blood is known as hematocrit. The hematocrit value is very important for early detection of several diseases. An appreciable increase or reduction of the hematocrit value might be a disease indication like anemia. In many cases, these diseases are not serious but they may cause other severe problems. Thus, an early detection might be vital $[16,15]$.

In this paper, a blood optical microscopy imaging analysis is performed by detecting the red blood cells in blood samples using the Hough transform and neural networks. The Hough transform technique provides the detection of each object or cell. This technique is frequently applied on image processing because of its efficacy and low complexity for the shape and feature detection. At present, it is useful in medical imaging studies [5, 13].

Neural networks have already been used for a wide variety of tasks in medicine, from medical imaging, signal processing to biomedical researches. In particular, multilayer perceptrons (MLP) are considered as universal approximators so they can be adapted to solve different real problems inside the field of medical imaging $[2,6,1]$. In this approach, the method classifies the detected cells between red blood cells and other elements such as white blood cells or spurious objects, among others. Recently, convolutional neural networks, which are an evolution of the MLP, have been successfully applied on image recognition and classification with higher accuracy rates than traditional techniques. A comparative between the two types of neural networks (MLP and CNN) is carried out.

The rest of the paper is structured as follows. Section 2 presents the methodology of the proposal, differentiating between the detection of blood cells and their subsequent classification. A complete suite of experiments is performed in Section 3, where both quantitative and qualitative results are showed over a dataset of blood images. Finally, our conclusions are provided in Section 4 .

\section{Methodology}

Our proposal can be divided into two steps: the detection of the red blood cells and their classification. An schema of our framework is represented in Figure 1. Section 2.1 describes how blood cells are detected inside the image regardless of their size. A classification process is performed from the detected cells in order to identify the actual red blood cells. This stage is detailed in Section 2.2.

\subsection{Red blood cells detection}

The first step is the detection of the possible red blood cells that appear in the image. For this purpose, we apply the Circle Hough Transform (CHT) to detect 


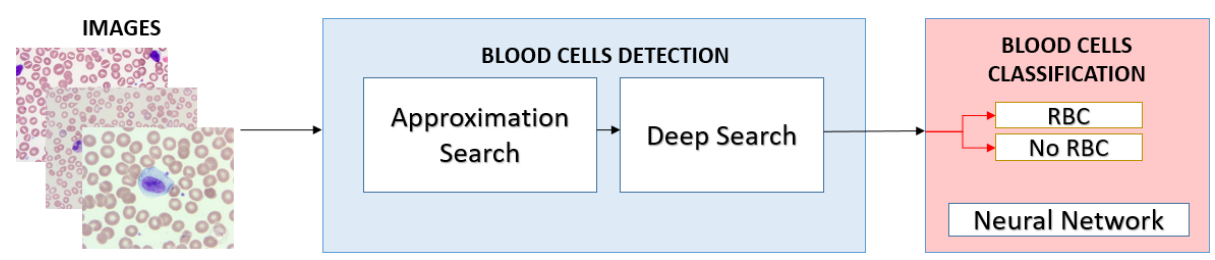

Fig. 1. Schema of the operation of the proposal

circular objects in the image. This is because the geometric shape of the red blood cells is similar to a circle.

The CHT is computed as follows. As known, the equation for a circle in two dimensions reads as follows:

$$
(x-a)^{2}+(y-b)^{2}=r^{2}
$$

where $(a, b)$ is the center of the circle, and $r>0$ is the radius. The circle Hough transform takes as input a set of $N$ edge points in the image:

$$
\mathcal{S}=\left\{\left(x_{i}, y_{i}\right) \in \mathbb{R}^{2} \mid i \in\{1, \ldots, N\}\right\}
$$

Then a score for each tentative circle, given by its three parameters $(a, b, r) \in$ $\mathbb{N}^{3}$, is computed as the count of edge points which lie in that circle. To that end, the parametric equation of the circle is considered:

$$
(x, y)=(a+r \cos \theta, b+r \sin \theta)
$$

After that the equation is rearranged as follows:

$$
(a, b)=(x+r \cos \theta, y+r \sin \theta)
$$

Finally, for each edge point in $\mathcal{S}$, each tentative value of the radius $r$, and each angle $\theta$, a vote is cast for the resulting tentative circle $(a, b, r)$, as given by (4). The tentative circles with the highest number of votes are declared as detected circles.

In order to have an approximation of the radii $r$ of the different blood cells that can be detected, a first search is accomplished because the scale of the image is unknown. The operation is to reduce the size of the image by applying a scale of 10 times (so that, the size in pixels of the image is reduced 100 times because the image is two-dimensional and the scale decreases each dimension size in 10 times). After that, a circles search is made in the reduced image using a radius rank between 2 and 10 pixels, counting the number of the detected circles with each of these radii.

The radius which presents the highest accumulation of circles will be selected to the second and deeper search. In this case, a new circle search by using the Circle Hough Transform is made again in the original image (not the reduced one). A rank around the most numerous radius previously found, increasing 
its size by 10 for adjusting to the original image, is applied as possible radius interval. In particular, a rank of difference 10 around the most numerous radius is defined.

\section{$2.2 \quad$ Red blood cells classification}

Once we have detected the blood cells, we need to classify them into red blood cells or other kind of blood cells. In this case we have considered two approaches to solve it.

The first predictive model is based on a basic neural network for classification tasks; specifically, the multilayer perceptron (MLP). This network consists of an input layer, where each input pattern is the median color of each detected cell (that is, it has three values which belong to the RGB value), a hidden layer with $M$ neurons and an output layer with one neuron whose value represents the probability of being red blood cell or not. The estimated value for the number of neurons in the hidden layer is $M=6$. It has been empirically checked that there is not much difference in the classification performance when this parameter increases.

The second analyzed model is the Alexnet convolutional neural network (CNN) [10]. Alexnet was developed by using several convolutional and fullyconnected layers and its aim was to classify the IMAGENET dataset ${ }^{1}$. The network inputs are the images associated to the blood cells detected.

In both cases, a balanced set of input samples has been considered to train the network, where only two classes are available: red blood cell or not. The images have been selected from the testing set of images and each one of them exhibits different blood cells. A dataset with all the images with the same dimension (227x227 pixels) is required to train the CNN. Nevertheless, the blood cell samples does not present this required size. Therefore, we have applied a resize process to solve this issue. Some different samples of these images can be found in Figure 2.

Since not all the images show the same scale in blood cells, it is needed to correct it and calculate the real size of cells. Hence, it is possible to estimate this value by knowing that the real size of the radius of a red blood cell is 3.9 micrometers approximately (an average diameter of 7.8 micrometers) [7].

\section{$3 \quad$ Experiments}

In this section, both the cell detection and cell classification approaches are applied to a set of blood sample images of heterogeneous sizes. In order to evaluate our methodology, a selected set of 10 images, which exhibit several blood cells and show different issues like overlapping objects, different illumination or scale in the image, is considered. They have been taken from the ASH Image

\footnotetext{
${ }^{1}$ http://www.image-net.org
} 


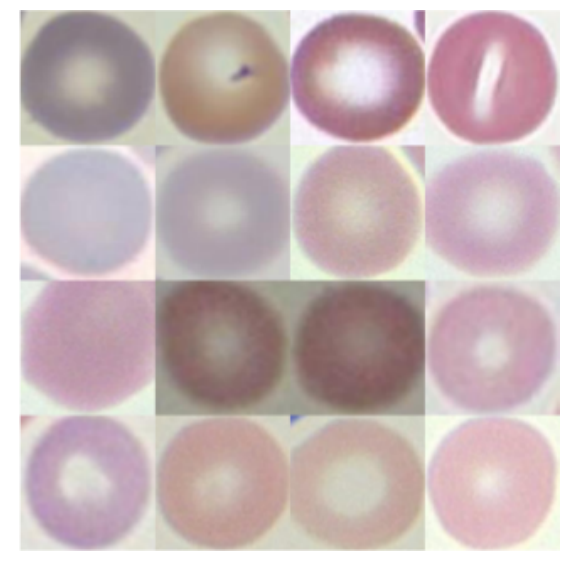

(a)

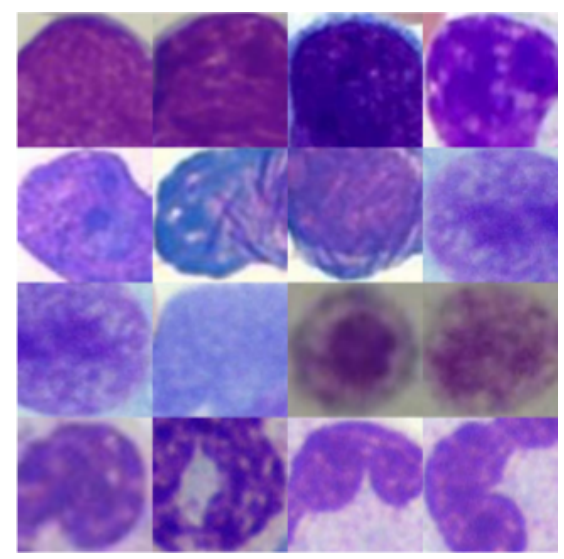

(b)

Fig. 2. Some samples of blood cell images used for the training process of the neural networks. The resize process is already applied on each displayed region. (a) exhibits red blood cells and (b) shows non red blood cells.

Bank $^{2}$. The selected images from this repository are named as follows: 1050, 1051, 1068, 1071, 1072, 60095, 60475, 60550,60802 and 60974.

In addition, we have selected several blood cell images from the used dataset and we have labeled them. This has to carry out in order to train the networks. Due to the low number of non red blood cells that are presented, we have increased this number with data augmentation techniques.

Furthermore, we have manually annotated the centroid of each red blood cell of each image to test the performance of the detection and classification of this kind of cells, using this information as a ground truth.

A robust performance of the goodness of the proposal is achieved due to a 5 -fold strategy is employed, where the 80 percent of the data form the training set and the remaining 20 percent comprise the test set. This process is repeated 10 times and each time the order of the images is randomly selected. Moreover, the training cell images corresponding to an image is randomly chosen too, with 20 red blood cell images and other 20 of non red blood cell images. After the training of the networks, the test set is used to measure the performance of the proposal.

Some different well-known measures have been selected in order to compare the performance of the detection and the classification of the blood cells from a quantitative point of view. In this work we have considered the spatial accuracy (S), the Accuracy (Acc) and the F-measure (Fm). All this measures provide values in the interval $[0,1]$, where higher is better, and represent the percentage of hits of the system.

\footnotetext{
${ }^{2}$ http://imagebank.hematology.org
} 


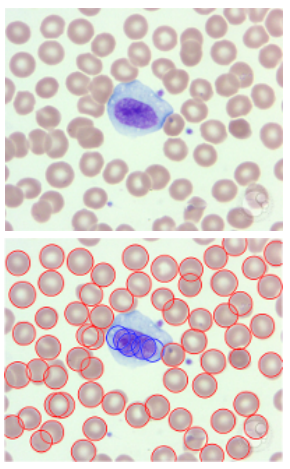

(a)

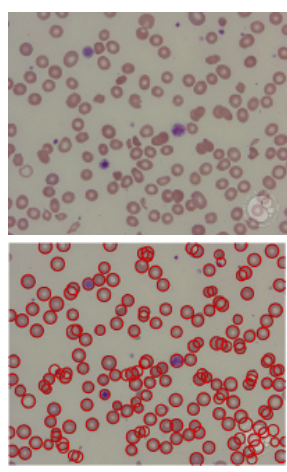

(b)

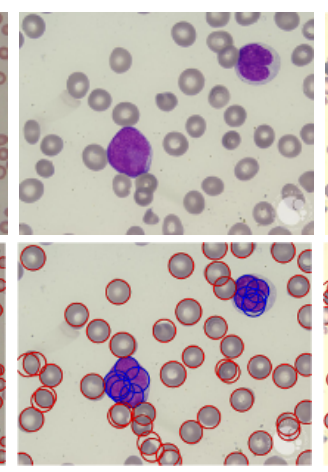

(c)

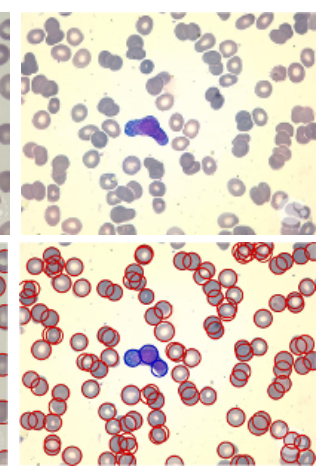

(d)

Fig. 3. Detection and classification of the red blood cells. First row exhibits the raw images and second row shows the result provided by the proposal. Each column presents an image of the tested dataset. Columns (a), (b), (c) and (d) corresponds to images 1050, 1068, 60095 and 60974, respectively. Each blood cell detected is indicated with a circle and the color shows its classification: red is for red blood cell and blue for non red blood cell.

True positives (TP), True negatives (TN), False negatives (FN), false positives (FP), the precision ( $\mathrm{PR}$ ), the recall (RC), the specificity (SP) and the percentage of wrong classifications (PWC) rates are also used in this work. Among all these measures, the spatial accuracy, the accuracy and the F-measure provide a good overall evaluation of the performance of a given method, while FN must be considered against FP (lower is better), and PR against RC (higher is better).

The definition of each measure can be described as follow:

$$
\begin{gathered}
S=\frac{T P}{T P+F N+F P} \quad A c c=\frac{T P+T N}{T P+F P+F N+T N} \\
R C=\frac{T P}{T P+F N} \quad P R=\frac{T P}{T P+F P} \quad S P=\frac{T N}{F P+T N} \\
F N R=\frac{F N}{T P+F N} \quad F P R=\frac{F P}{F P+T N} \\
\text { F-measure }=2 * \frac{P R * R C}{P R+R C} \\
P W C=100 * \frac{F N+F P}{T P+F P+T N+F N}
\end{gathered}
$$

In order to test the performance of the detection of red blood cells we have compared the centroids of the ground truth against the centroids of the estimated blood cell. Because we can estimate the scale of the test image and it is well-known that the radius of a red blood cell is 3.9 micrometers [7], we have considered that an estimated centroid is correctly detected if it has a distance 
Table 1. Performance of the detection of red blood cells. First column indicates the image from the dataset and remaining columns show the yielded mean performance achieved by the proposal in different measures. Each row represents a tested image from the dataset and its mean performance, and last row exhibits the average of the values of each measure.

\begin{tabular}{c|ccc|ccccc}
\hline Image & TP & FP & FN & RC & FNR & PR & Fm & S \\
\hline 1050 & 63 & 7 & 2 & 0.969 & 0.031 & 0.900 & 0.933 & 0.875 \\
1051 & 70 & 9 & 26 & 0.729 & 0.271 & 0.886 & 0.800 & 0.667 \\
1068 & 129 & 50 & 12 & 0.915 & 0.085 & 0.721 & 0.806 & 0.675 \\
1071 & 55 & 10 & 7 & 0.887 & 0.113 & 0.846 & 0.866 & 0.764 \\
1072 & 52 & 11 & 4 & 0.929 & 0.071 & 0.825 & 0.874 & 0.776 \\
60095 & 41 & 18 & 1 & 0.976 & 0.024 & 0.695 & 0.812 & 0.683 \\
60475 & 199 & 32 & 11 & 0.948 & 0.052 & 0.861 & 0.902 & 0.822 \\
60550 & 82 & 40 & 6 & 0.932 & 0.068 & 0.672 & 0.781 & 0.641 \\
60802 & 77 & 24 & 4 & 0.951 & 0.049 & 0.762 & 0.846 & 0.733 \\
60974 & 97 & 27 & 5 & 0.951 & 0.049 & 0.782 & 0.858 & 0.752 \\
\hline Average & & & & 0.919 & 0.081 & 0.795 & 0.848 & 0.739 \\
\hline
\end{tabular}

of 1.5 micrometers from the ground truth centroid. Furthermore, we only have considered those ground truth centroids that appear inside the image. According to this restrictions, the detection test does not consider the TN measure. On the other hand, the test of the performance of the classification is over the detected blood cells.

From a qualitative point of view, our proposal exhibits a good performance in the detection and the classification of the red blood cells. This information can be observed in Figure 3. It can be appreciated how the proposal considers several blood cells as red ones in the detection step. Nevertheless, after the application of the classification process, the proposal corrects these predictions. It must also be highlighted the detection of the overlapping blood cells or those that appear in the border of the image. Another problem is shown when the proposal detects two (or more) centroids for only one red blood cell.

On the other hand, from a quantitative point of view. The performance of the detection of our proposal can be observed in Table 1. The average of blood cell detection is around 0.74, mainly because the CHT not only detects red blood cells, but also any circular element that is present in the image. Thus, it is possible to detect several red blood cells in the location of larger white blood cells, so it is essential to have a posterior stage that correctly detects or classifies those circles as red blood cells.

The performance of the two proposed classification model is shown in Tables 2 and 3. We compare the accuracy of these two models, as we can see in Figure 4. Although both models obtain very good red cell classification rates, in general, the CNN proposal slightly improves the generated results by the standard neural network. This happens because by providing more input information (the complete image of each detected circle instead of a vector of three elements with 
Table 2. Performance of the classification of red blood cells by employing the basic neural network. First column indicates the image from the dataset and remaining columns show the yielded mean performance achieved by the proposal in different measures. Each row represents a tested image from the dataset and its mean performance, and last row exhibits the average of the values of each measure.

\begin{tabular}{c|ccccccccccccc}
\hline Image & TP & TN & FP & FN & RC & SP & FPR & FNR & PWC & PR & Fm & S & Acc \\
\hline 1050 & 65 & 5 & 0 & 0 & 1.00 & 1.000 & 0.000 & 0.00 & 0.000 & 1.000 & 1.000 & 1.000 & 1.000 \\
1051 & 70 & 6 & 3 & 0 & 1.00 & 0.667 & 0.333 & 0.00 & 3.797 & 0.959 & 0.979 & 0.959 & 0.962 \\
1068 & 138 & 0.4 & 42.6 & 0 & 1.00 & 0.009 & 0.991 & 0.00 & 23.536 & 0.764 & 0.866 & 0.764 & 0.765 \\
1071 & 56 & 6 & 3 & 0 & 1.00 & 0.667 & 0.333 & 0.00 & 4.615 & 0.949 & 0.974 & 0.949 & 0.954 \\
1072 & 57 & 3.3 & 2.7 & 0 & 1.00 & 0.550 & 0.450 & 0.00 & 4.286 & 0.955 & 0.977 & 0.955 & 0.957 \\
60095 & 48 & 11 & 2 & 0 & 1.00 & 0.846 & 0.154 & 0.00 & 3.279 & 0.960 & 0.980 & 0.960 & 0.967 \\
60475 & 225 & 2 & 7 & 0 & 1.00 & 0.222 & 0.778 & 0.00 & 2.991 & 0.970 & 0.985 & 0.970 & $\mathbf{0 . 9 7 0}$ \\
60550 & 86 & 30.8 & 7.2 & 0 & 1.00 & 0.811 & 0.189 & 0.00 & 5.806 & 0.923 & 0.960 & 0.923 & 0.942 \\
60802 & 84 & 11 & 7 & 0 & 1.00 & 0.611 & 0.389 & 0.00 & 6.863 & 0.923 & 0.960 & 0.923 & $\mathbf{0 . 9 3 1}$ \\
60974 & 106 & 3 & 17 & 0 & 1.00 & 0.150 & 0.850 & 0.00 & 13.492 & 0.862 & 0.926 & 0.862 & $\mathbf{0 . 8 6 5}$ \\
\hline Average & & & & & 1.00 & 0.553 & 0.447 & 0.00 & 6.867 & 0.926 & 0.961 & 0.926 & 0.931 \\
\hline
\end{tabular}

Table 3. Performance of the classification of red blood cells by employing the convolutional neural network. First column indicates the image from the dataset and remaining columns show the yielded mean performance achieved by the proposal in different measures. Each row represents a tested image from the dataset and its mean performance, and last row exhibits the average of the values of each measure.

\begin{tabular}{c|cccc|ccccccccc}
\hline Image & TP & TN & FP & FN & RC & SP & FPR & FNR & PWC & PR & Fm & S & Acc \\
\hline 1050 & 65 & 5 & 0 & 0 & 1.000 & 1.000 & 0.000 & 0.000 & 0.000 & 1.000 & 1.000 & 1.000 & 1.000 \\
1051 & 70 & 6 & 3 & 0 & 1.000 & 0.667 & 0.333 & 0.000 & 3.797 & 0.959 & 0.979 & 0.959 & 0.962 \\
1068 & 136.9 & 3.8 & 39.2 & 1.1 & 0.992 & 0.088 & 0.912 & 0.008 & 22.265 & 0.777 & 0.872 & 0.773 & $\mathbf{0 . 7 7 7}$ \\
1071 & 56 & 7 & 2 & 0 & 1.000 & 0.778 & 0.222 & 0.000 & 3.077 & 0.966 & 0.982 & 0.966 & $\mathbf{0 . 9 6 9}$ \\
1072 & 57 & 5 & 1 & 0 & 1.000 & 0.833 & 0.167 & 0.000 & 1.587 & 0.983 & 0.991 & 0.983 & $\mathbf{0 . 9 8 4}$ \\
60095 & 48 & 11 & 2 & 0 & 1.000 & 0.846 & 0.154 & 0.000 & 3.279 & 0.960 & 0.980 & 0.960 & 0.967 \\
60475 & 223.2 & 2 & 7 & 1.8 & 0.992 & 0.222 & 0.778 & 0.008 & 3.761 & 0.970 & 0.981 & 0.962 & 0.962 \\
60550 & 86 & 31 & 7 & 0 & 1.000 & 0.816 & 0.184 & 0.000 & 5.645 & 0.925 & 0.961 & 0.925 & $\mathbf{0 . 9 4 4}$ \\
60802 & 83.2 & 11 & 7 & 0.8 & 0.990 & 0.611 & 0.389 & 0.010 & 7.647 & 0.922 & 0.955 & 0.914 & 0.924 \\
60974 & 105.7 & 3.1 & 16.9 & 0.3 & 0.997 & 0.155 & 0.845 & 0.003 & 13.651 & 0.862 & 0.925 & 0.860 & 0.863 \\
\hline Average & \multicolumn{1}{|c|}{} & & 0.997 & 0.602 & 0.398 & 0.003 & 6.471 & 0.932 & 0.963 & 0.930 & $\mathbf{0 . 9 3 5}$ \\
\hline
\end{tabular}

the medium color) the $\mathrm{CNN}$ can better discern which circles correspond to the red blood cells, despite the considerable increase of complexity of this second proposal. Possibly, the development of CNNs adapted to this specific issue manages to improve the classification result in images with smaller cell sizes (image 60974) and with low illumination (image 1068).

\section{Conclusion}

In this work, a methodology for the detection of red blood cells in medical images is presented, based on the use of the circle Hough transform (CHT) for detection 


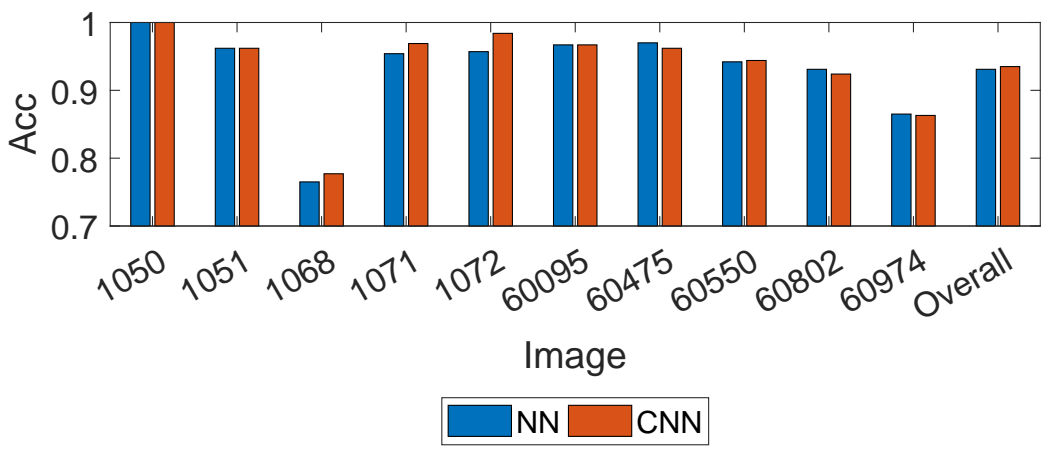

Fig. 4. Accuracy comparison between the performance achieved by the basic neural network and the convolutional neural network in each image from the dataset. The bars of the overall represent the obtained average accuracy.

and neural networks for classification. This classification module is necessary since not all the detected circles correspond to red blood cells, which implies an identification process to ensure which circle corresponds to a red blood cell or not. Specifically, two alternatives have been tested for this classification, a standard neuronal network (multilayer perceptron, MLP) and a convolutional neuronal network $(\mathrm{CNN})$. A set of heterogeneous medical images has been used for the evaluation of the different proposals. It is observed that the results of the first detection module are remarkable, with success rates close to $75 \%$, while the classification rates are close to $94 \%$ on average for both CNN and MLP. Although there is some improvement in favor of the CNNs, the implementation of ad hoc CNNs associated with this issue could still improve the results obtained.

\section{References}

1. Baxt, W.G.: Application of artificial neural networks to clinical medicine. The lancet 346(8983), 1135-1138 (1995)

2. Clark, J.: Neural network modelling. Physics in Medicine and Biology 36(10), 1259 (1991)

3. Cornbleet, J.: Spurious results from automated hematology cell counters. Laboratory Medicine 14(8), 509-514 (2016)

4. Davis, R., Boyers, S.: The role of digital image analysis in reproductive biology and medicine. Archives of pathology \& laboratory medicine 116(4), 351-363 (1992)

5. Ecabert, O., Thiran, J.P.: Adaptive hough transform for the detection of natural shapes under weak affine transformations. Pattern Recognition Letters 25(12), 1411-1419 (2004) 
6. Egbert, D.D., Kaburlasos, V.G., Goodman, P.H.: Neural network discrimination of subtle image patterns. In: Neural Networks, 1990., 1990 IJCNN International Joint Conference on, pp. 517-524. IEEE (1990)

7. Fedosov, D.A., Caswell, B., Karniadakis, G.E.: A multiscale red blood cell model with accurate mechanics, rheology, and dynamics. Biophysical journal 98(10), 2215-2225 (2010)

8. Imeri, F., Herklotz, R., Risch, L., Arbetsleitner, C., Zerlauth, M., Risch, G.M., Huber, A.R.: Stability of hematological analytes depends on the hematology analyser used: a stability study with bayer advia 120, beckman coulter lh 750 and sysmex xe 2100. Clinica chimica acta 397(1), 68-71 (2008)

9. Krause, J.: Automated differentials in the hematology laboratory. American journal of clinical pathology 93(4 Suppl 1), S11-6 (1990)

10. Krizhevsky, A., Sutskever, I., Hinton, G.: ImageNet classification with deep convolutional neural networks. Advances in Neural Information Processing Systems 25, 1097-1105 (2012)

11. Lantis, K.L., Harris, R.J., Davis, G., Renner, N., Finn, W.G.: Elimination of instrument-driven reflex manual differential leukocyte counts: optimization of manual blood smear review criteria in a high-volume automated hematology laboratory. American journal of clinical pathology 119(5), 656-662 (2003)

12. McAuliffe, M.J., Lalonde, F.M., McGarry, D., Gandler, W., Csaky, K., Trus, B.L.: Medical image processing, analysis and visualization in clinical research. In: Computer-Based Medical Systems, 2001. CBMS 2001. Proceedings. 14th IEEE Symposium on, pp. 381-386. IEEE (2001)

13. Philip, K.P., Dove, E.L., McPherson, D.D., Gotteiner, N.L., Stanford, W., Chandran, K.B.: The fuzzy hough transform-feature extraction in medical images. IEEE Transactions on Medical Imaging 13(2), 235-240 (1994)

14. Schmitt, J.M., Zhou, G.X., Miller, J.: Measurement of blood hematocrit by dualwavelength near-ir photoplethysmography. In: Physiological monitoring and early detection diagnostic methods, vol. 1641, pp. 150-162. International Society for Optics and Photonics (1992)

15. Tang, Z., Lee, J.H., Louie, R.F., Kost, G.J.: Effects of different hematocrit levels on glucose measurements with handheld meters for point-of-care testing. Archives of pathology \& laboratory medicine 124(8), 1135-1140 (2000)

16. Wennecke, G.: Hematocrit-a review of different analytical methods. Radiometer Medical ApS (2004) 